\title{
Distributed Cognition and Mobile Healthcare Work
}

\author{
Joseph McKnight \\ Department of Computer Science, \\ Trinity College Dublin, Ireland \\ mcknigjg@cs.tcd.ie
}

\author{
Gavin Doherty \\ Department of Computer Science, \\ Trinity College Dublin, Ireland \\ Gavin.Doherty@cs.tcd.ie
}

\begin{abstract}
In this paper, we look at the application of the DiCoT methodology [4] to the analysis of a mobile healthcare setting. While the methodology includes a set of principles and themes, and provides significant leverage in the analysis of control room settings, the highly mobile nature of healthcare work throws up some unique challenges. We present an analysis of patient process management within a hospital using DiCoT. While the information flow and artefact analysis themes of the methodology were found to be useful in the analysis, the mobile nature of the work meant that the principles outlined for the physical theme only partially cover the issues of interest.
\end{abstract}

\section{INTRODUCTION}

When designing technology to support complex work, it is important to build up an understanding of the work as it is currently carried out. This understanding can help to establish requirements, can provide input to the design, and can help in reasoning about the impact of a proposed system. Analytic frameworks can help to bridge the gap between data from observational studies and the design process. Distributed Cognition [8][9] has been put forward as a framework which can help us to understand cognition as it occurs in the workplace [2][5][11]; however in itself, it does not provide a methodology for its application to design. DiCoT [4] is a methodology developed for supporting analysis of distributed cognition in small teams and is based on core ideas from the distributed cognition literature. The methodology is based around three main themes, physical layout, information flow and artefacts. It has been applied to an ambulance control centre [6] and XP programming teams [13]; however the overall scope of the method is still unclear.

In this paper we use DiCoT to analyse the work of processing cancer patients who are being assessed for surgery. Healthcare environments are characterised by a high degree of cooperation and mobility, and so a method based around Distributed Cognition appears to be a promising candidate for analysis. While the challenges of the healthcare environment are such that we cannot expect complete coverage from any single analysis approach, the methodology may provide a useful starting point.

\section{MOBILE HEALTHCARE WORK}

The applicability of the Distributed Cognition framework in healthcare has previously been discussed in [11], who use Distributed Cognition as a guiding theme in analysis of

(C) The Author 2008.

Published by the British Computer Society cognitive artefacts [12] such as schedules, worksheets and display boards and their use by clinicians in a healthcare environment. They show the power of analysing these artefacts from a Distributed Cognition perspective in uncovering the work that the artefacts are designed to support so appropriate redesign can be attempted. Distributed cognition has also been applied to tracing information flow to analyse and predict where information breakdown can occur in clinical systems [7] using the DIB (Determining Information flow Breakdown) method

The environment is one subject to continuous change (evolving best practice, regulatory changes, re-organisation), and as a result of this, there are continuously changing demands placed on the information systems. A well defined method for analysis which can be applied repeatedly, with a consistent quality of output is desirable in such a situation. Given recent technology advances in mobile computing, and the nature of the work, in the coming years it is to be expected that there will be many mobile technology development projects within the healthcare domain, and it would be desirable to have an analytic framework which is appropriate for the needs of such projects.

2.1 Case study: patient process management The case study concerns a large cancer treatment healthcare facility, where the cardio-thoracic surgery unit receives 5 to 10 new case referrals per week. A study was performed with the relevant clinicians and administrative staff at the hospital. The study is based on semi-structured interviews with clinicians, examination of paper-based artefacts, and observations of a number of key locations within the working environment. A previous and highly detailed ethnographic study of the multidisciplinary team meeting (MDTM), which is central to the process, was also of much benefit in understanding these activities [10].

Each new referral must be processed in order to determine whether the patient is suitable for surgery. This involves gathering the information needed to discuss the patient at the MDTM, which stages (classifies) the patient's cancer, followed by an outpatient assessment before deciding if surgery is required, followed then by the surgery itself and aftercare. Given the large number of specialist staff who collaborate through the MDTM, it is vital that the necessary tests have been carried out and reviewed prior to the meeting. The work of managing the patients through the process is mainly carried out by the consultant cardio-thoracic surgeon and specialist oncology coordinator, aided by administrative staff.

The processing of patients is tracked by various paper based artefacts that are maintained by the specialist oncology coordinator. The artefacts used include paper notebooks with the most relevant patient information, lists of patients in treatment with status, and a file with all documents related to the patient. This is necessary because patients in process are not physically located at the hospital ward (or technically a patient of the hospital) until outpatient assessment or surgery. Even though patient treatment is managed from the main hospital, 
scans and tests may be carried out by proxy at the referring hospital. As a result, the system is highly dependent on the oncology coordinator and paper based artefacts to function. For example, a patients' processing may be on hold until a scan is completed at another hospital or a test result becomes available. Unless these actions are followed up by the oncology coordinator, using paper files, notebook and various artefacts, a delay in processing could result, with potentially serious consequences. While the use of a paper-based system may seem atypical, the constant evolution of processes means that often workers have to fall back on ad-hoc methods while they wait for formal structures and technological support to emerge.

\section{AN ANALYSIS USING DiCoT}

\subsection{Physical layout}

As the clinicians are mobile they use a number of locations for work; these are typically shared-use rather than exclusive areas. The consultant and coordinator meet regularly in the angiogram or "angio" room, which is a common space used by the clinicians close to the operating theatres, to discuss patient cases. Once per week the consultant and coordinator attend an MDTM at a special facility built to accommodate such meetings to clinically stage patient cancers. Patient assessment is carried out by the coordinator either at the surgery ward or outpatient clinic on a one-to-one basis to assess the general health of patients pre-surgery.

DiCoT provides a number of analysis principles concerning physical layout, for example:

Space and Cognition: Observations from the angiogram room show how awareness of new case arrivals is picked up by A4 sized envelopes on the desk (often annotated with sticky notes). Members of the surgery team often browse these files before the arrival of the consultant and coordinator. This also plays a role in supporting situation awareness.

Situation Awareness: As the surgery team constantly uses the angiogram room to view patient scans on the Hospital Information System (HIS), they can overhear discussions between the consultant and coordinator. They would hear case discussion, surgery planning and other process related information. The MDTM also plays a large role in maintaining situation awareness, but this is a complex setting in itself and analysis is beyond the scope of this paper.

Horizon of Observation: The use of the angio room to view scans while meetings take place can be seen as impacting on the horizon of observation of the team. However, the information transferred in this way is incomplete and haphazard. For this case study it seems to be a principle of more interest when we move towards design.

The remaining 5 principles from the physical layout theme are less immediately useful for our analysis mostly due to the mobile nature of work. It could be argued that appropriate technology in locations such as the angiogram room could better support the activities of the team, in which case more of the principles would come in to play in discussing the impact of such changes.

\subsection{Information Flow}

As the job title suggests the oncology coordinator is the main source and sink of information related to processing patients. Therefore the coordinator is central to the operation of the

\footnotetext{
* The name derives from historic rather than current usage.
}

system, and buffers and propagates the overall information flow as required. This is evidenced by the role of the coordinator in patient case discussions with the consultant and at the MDTM.

Patient case discussion meetings between the coordinator and consultant occur twice weekly in the angiogram room. At these meetings the coordinator and consultant decide on the next steps to be taken regarding each patient. This could be surgery, MDTM discussion, requesting scans, or outpatient assessment. It will also involve discussion of the results of previous actions taken regarding patients. The meeting determines which patient cases are to be pursued next; in effect they become active in the work of the coordinator, unlike patients who are waiting for a scan or test result to arrive. At the MDTM the consultant will present patient cases to the team while being assisted by the coordinator who has prepared the information. The list of patients to be discussed will have been agreed at a previous patient case discussion meeting. Discussion at the MDTM results in a patients cancer being clinically staged, which is a vital piece of information to decide on appropriate action. The outcome of these discussions will play a role in the immediate work related to a patient. Information movement: The movement of information around the cognitive system is primarily physical (paper artefacts carried by the coordinator) and face to face. While the coordinator gathers processing related information, this is transferred face to face with the consultant. Likewise they perform a similar transfer of information to the MDTM members before case discussions. When a patient completes scans at outside hospitals they must sent to the local hospital for review by the local radiologist, whose report becomes available on the HIS. The report will be viewed by the consultant pre MDTM and discussed at the MDTM with team members.

Information Transformation: Before patients are discussed at the MDTM they must complete a series of tests and scans. The dates and the fact that a test or scan has been completed become processing information for the coordinator and are stored in this format in a notebook of patient's status details. Scans may arrive from outside hospitals in the patients file but the consultant will be mainly interested in the radiologists report on the scan. The stream of information on test results and scans is transformed by the coordinator into status information eg. that the patient case is ready for discussion at MDTM.

Information Hubs: We can view the MDTM and patient case discussion meetings as information hubs. At these meetings different sources of patient processing and medical information are used to decide future actions for patients. The processing information is provided by the coordinator while the medical information such as scans and reports are available on the HIS.

Buffers: The coordinator is the main buffer in the system as patient information is held by the coordinator until case discussion with the consultant.

Communication Bandwidth: As communication in the system is mostly through face to face meetings the communication bandwidth is high. However as some patients are processed by proxy at other hospitals communication here can be limited to electronic communication (including videoconference at MDTM) or telephone calls.

Informal and Formal Communication: As the coordinator is in periodic contact with patients via phone calls and outpatient assessment, the coordinator has information none of the other clinicians have. This knowledge is sometimes used in borderline surgery cases. For example, the coordinator in discussion with the surgeon might mention that the general 
health of a patient was fine up until very recently, while outpatient assessment data of general health might indicate that the patient was a borderline case for surgery.

Behavioural Trigger Factors: The members of the team have limited visibility on the overall status regarding patients across different stages of the process. Discussion of cases at the MDTM, surgical schedules and patients on the ward can be seen as the drivers for different members of the team.

The meetings between co-ordinator and consultant are obviously key to the operation of the system. We can see these meetings as a process of inter-subjective space negotiation between the coordinator and consultant. As they are non colocated distributed mobile workers they must ensure they have the same understanding of the patient's previous processing history before discussions proceed on future actions. The discussions at the MDTM would be analogous to this process also. We would expect that this type of inter-subjective space negotiation to be more prevalent in healthcare than many other domains as work is characterized by mobility and distribution of workers [3].

\subsection{Artefact Analysis}

The coordinator uses a notebook that contains the most up to date information relating to patient processing and their status. The notebook is used because it is easily portable and it contains a concise, personally tailored and quickly referenced summary of the patients processing history. This is non medical information such as scans completed and dates. The coordinator also produces lists of patients that will be discussed at patient case discussion meetings and MDTM.

Mediating Artefacts: The notebook containing a summary of patient processing information is used by the coordinator in patient case discussions. It is used to quickly relay processing information to the consultant or when answering queries from patients or other hospitals.

Creating Scaffolding: The patient lists created by the coordinator are used to guide discussion of patients at meetings. By creating these lists the coordinator is ensuring that no patients' case is omitted from discussion and relieves the coordinator of the burden of remembering cases to be discussed.

Co-ordination of Resources: A full resource-based analysis [5] is beyond the scope of this paper, but the concept appears to be very applicable; in particular the paper artefacts used by the coordinator provide information which can be characterised as plan, status and history type resources. It can also be seen that information resources are inadequate for supporting certain goals relevant to patient process management.

It can be seen that many personal paper based artefacts exist in the cognitive system which make it very vulnerable if artefacts are misplaced, or if changes to staffing occur. The movement of these artefacts is also necessary in order that the information that they contain can be transferred to the consultant or MDTM team.

Following initial analysis, the Representation-Goal Parity principle appears to be more relevant to analysis of proposed technological support than to understanding the system as-is.

\subsection{Evolution over Time}

In [13] a number of further principles are listed which although not in the original method, appear to be very relevant to this domain.

Cultural heritage: The "angiogram" room used by the consultant and coordinator for patient case discussions was originally used to view angiograms. As it no longer serves this purpose it is now used as a general meeting area and space where surgeons come to view patient scans and reports on the HIS. It is located in an area just outside the (restricted) surgery theatres, which makes it an ideal place where surgeons, other clinicians and non clinical staff can meet for impromptu discussions.

As with many case studies in the medical area, it can also be seen that the format and use of personal paper-based artefacts for patient process management is something which has evolved over time. A particular vulnerability within the system is that the knowledge embedded within these could be lost with changes to staffing or to the organisational structure.

The shift towards use of MDTM has also resulted in significant changes in the culture of the organisation, and an emerging set of norms and working practices which have significant impact on patient process management.

Expert Coupling: As expected in healthcare each specialty has a specific role in the system. However the role played by the coordinator is very specific, while being extremely experienced in the role. It is the coordinators "unwritten" role to manage the processing of patients as autonomously as possible from the consultant, only involving the consultant when necessary. The system currently operates with this level of autonomy but this is due to the trust and knowledge in the relationship between the consultant and coordinator that has built up over time.

\subsection{Social Structures}

Social Structure and Goal Structure: The social structure and goal structure are difficult to co-analyse in the case study. The consultant and coordinator work together in an autonomous fashion as discussed, but both have the same goal in processing patients, and maintaining the safety and performance of the overall system (patients do not wait overlong at any stage of the process, required information is obtained, scans and tests are followed up on). The MDTM members however are transiently responsible for clinically staging a patients' cancer, which is their goal at the meeting.

Socially Distributed properties of Cognition: The MDTM members in conjunction with the consultant come to a decision on the staging of a patient's cancer. They use outside sources of information in this decision, such as the radiologists report. Similarly, when the consultant and coordinator meet to discuss patient cases they decide together on the appropriate course of action for a patient.

\section{DISCUSSION}

Some of the principles from the physical theme could not be utilised in our analysis due mainly to the mobile nature of the work. As the angiogram room is not dedicated or constructed to support the meetings that occur there, and is only used on a temporary basis, some of the principles derived from control room studies were less relevant to understanding the system as it operates at the moment.

The information flow theme however proved very powerful as all the principles proved useful in analysing the case study. One aspect of the dynamic of information flow we felt was not captured was the construction of a shared understanding of the situation preceding patient case discussion. We can see this as not just establishing common ground, but negotiation of an inter-subjective space [1], as control over what grounding takes place is an important part of this activity. This activity is again related to the mobile aspect of the clinicians work. As they are not collocated and meet periodically this initial negotiation 
must occur. We can also see this as relevant to analysis of the evolution of the system over time.

The artefact analysis also proved very relevant in our analysis. The fact that the Representation-Goal Parity principle was less applicable to the system as-is should be interpreted as indicating that better support is needed rather than the principle not being relevant. Likewise if support was implemented for planed actions for patients and meetings, it would be beneficial to analyse the proposed solution in terms of support for Coordination of Resources.

While space does not permit inclusion of a claims analysis of proposed design features (part of the methodology), it is clear from our analysis using DiCoT that a number of design considerations and possibilities can be extracted. The most obvious is the need for some representation of the current state of the system and the goal state. While this could be supported on a mobile device, another possibility would be to supplement this with a large shared display in the angiogram room that could show the patients currently in process for surgery, awaiting MDTM discussion and outpatient assessment. This would enable the coordinator and surgeon to have an external representation of the state of patient processing when discussing patients, but would also increase the situation awareness of the other members of the surgery team.

The plans for action on patients decided at the angiogram room meetings must be completed by the coordinator and could be supported by appropriate mobile technology. Similarly the MDTM can create numerous follow up actions for the coordinator. While we already know that the coordinator creates patient lists for patients that will be discussed at meetings if we combine management of these with support for planning we can help the coordinator in managing resources to support work processes. If this support was provided on a mobile device then the coordinator could dynamically work with the lists and plans as required, at any location.

\section{CONCLUSIONS}

Healthcare is an important area which throws up a number of unique challenges. While there are many issues and difficulties surrounding the use of mobile technology, due to the mobile nature of the work it seems inevitable that in the future many aspects of the work will be supported by personal mobile devices. It is worth remembering however that technology is not the only driver of change; the move towards MDT meetings is a clear example of this.

From our case-study analysis using DiCoT it was found that the Physical space theme provided useful but limited support for understanding the existing system due to the mobile nature of healthcare work and the transient use of locations to carry out work. However, it could prove more useful for reasoning about technology within certain locations - for example if a number of key locations were to be augmented with increased technological support. It could be that other physical principles should be developed to support the analysis - for example, to investigate the coupling of artefacts and activites across locations, and the physical movement of artefacts. The Information flow theme provided good analytic power, as all principles were found to be relevant. However we did identify a characteristic from our mobile workers, namely the need to negotiate an inter-subjective space preceding patient case discussions, which goes beyond situation awareness, and is more concerned with common ground between team members. The Artefact analysis also proved very relevant, but each issue within the analysis is substantial within its own right, and could be elaborated further within the healthcare domain. Within the artefact theme the lack of applicability of the RepresentationGoal Parity principle was seen as indicating an opportunity for improved support within the system. The Coordination of Resources principle can be used to identify shortcomings (inadequately resourced activities), but could also be useful for reasoning about features of proposed designs. Social structures and evolution of the system have already been identified as areas requiring expansion within the method, but still proved very relevant to the case study analysis.

Overall the methodology was found to be very useful in analysis of the case study; future work will explore the analysis of proposed design features.

\section{ACKNOWLEDGMENTS}

J. McKnight is supported by an Intel/IRCSET Enterprise Partnership. The authors would also like to thank staff at St. James Hospital for their support.

\section{REFERENCES}

[1] Alterman, R. (2007). "Representation, Interaction and Intersubjectivity”, Cognitive Science 31(5): 815-841.

[2] Artman, H. and Y. Waern (1999). "Distributed Cognition in an emergency Co-ordination Center." Cognition Technology and Work (1): 237-246.

[3] Bardram, J. and C. Bossen (2003). Moving to get ahead: Local Mobility and Collaborative Work. European Conference on Computer Supported Cooperative Work. Helsinki, Finland.

[4] Blanford, A. and D. Furniss (2005). "DiCoT: A methodology for applying Distributed Cognition to the design of team working systems." Interactive Systems: Design, Specification and Verification, Springer LNCS vol. 3941.

[5] Fields, R. P., P. M. Wright, et al. (1998). Air traffic control as a distributed cognitive system: a case study of external representations. Proceedings of the Ninth European Conference on Cognitive Ergonomics.

[6] Furniss, D. and Blandford, A. (2005) "Understanding Emergency Medical Dispatch in terms of Distributed Cognition: a case study", Ergonomics 49(12-13): 11741203.

[7] Galliers, J., S. Wilson and J. Fone (2004). A Method for Determining Information Flow Breakdown in Clinical Systems. IT in Healthcare. Portland Oregon, USA., International Journal of Medical Informatics.

[8] Hutchins, E. (1995). "How a Cockpit Remembers Its Speeds." Cognitive Science(19): 265-288.

[9] Hutchins, E. (1996). Cognition in the Wild, MIT Press.

[10] Kane, B. and Luz, S. (2006). Multidisciplinary medical team meetings: An Analysis of Collaborative Working with Special Attention to timing and Teleconferencing. Computer Supported Cooperative Work 15(5):501-535.

[11] Nemeth, C., R. Cook, P., O' Connor, M., Allan, P.(2004). "Using Cognitive Artifacts to Understand Distributed Cognition." IEEE Transactions on Systems, Man and Cybernetics 34(6).

[12] Norman, D. A. (1991). Cognitive Artefacts. In Carroll (ED). Designing Interaction: Psychology at The Human Computer Interface. Cambridge, pp.17-38.

[13] Sharp, H., Robinson, H., Segal, J., Furniss D. "The Role of Story Cards and the Wall in XP teams: A Distributed Cognition Perspective", pp. 65-75, AGILE 2006, 2006. 